\title{
Chapter 20 \\ Role of PACAP and VIP Signalling \\ in Regulation of Chondrogenesis and Osteogenesis
}

\author{
Tamas Juhasz, Andrea Tamas, Roza Zakany, and Dora Reglodi
}

\begin{abstract}
Pituitary adenylate cyclase activating polypeptide (PACAP) and vasoactive intestinal peptide (VIP) are multifunctional proteins that can regulate diverse physiological processes. These are also regarded as neurotrophic and antiinflammatory substances in the CNS, and PACAP is reported to prevent harmful effects of oxidative stress. In the last decade more and more data accumulated on the similar function of PACAP in various tissues, but its cartilage- and bone-related presence and functions have not been widely investigated yet. In this summary we plan to verify the presence and function of PACAP and VIP signalling tool kit during cartilage differentiation and bone formation. We give evidence about the protective function of PACAP in cartilage regeneration with oxidative or mechanically stress and also with the modulation of PACAP signalling in vitro in osteogenic cells. Our observations imply the therapeutic perspective that PACAP might be applicable as a natural agent exerting protecting effect during joint inflammation and/or may promote cartilage regeneration during degenerative diseases of articular cartilage.
\end{abstract}

Keywords $\mathrm{PKA} \bullet$ Mechanical stress $\bullet$ Hedgehog $\bullet$ BMP $\bullet$ Runx $2 \bullet$ Oxidative stress

\section{Abbreviations}

ALP Alkaline phosphatase

BMP Bone morphogenetic protein

cAMP Cyclic adenosine monophosphate

T. Juhasz $(\triangle) \cdot$ R. Zakany

Department of Anatomy, Histology and Embryology, Faculty of Medicine,

University of Debrecen, Nagyerdei krt. 98, Debrecen 4032, Hungary

e-mail: juhaszt@anat.med.unideb.hu

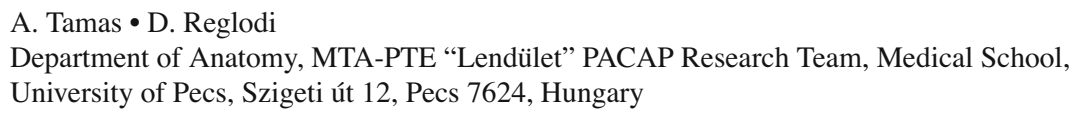

A. Tamas $\bullet$ D. Reglodi

Department of Anatomy, MTA-PTE "Lendület” PACAP Research Team, Medical School,

University of Pecs, Szigeti út 12, Pecs 7624, Hungary

D. Reglodi, A. Tamas (eds.), Pituitary Adenylate Cyclase Activating

Polypeptide - PACAP, Current Topics in Neurotoxicity 11,

DOI 10.1007/978-3-319-35135-3_20 
CREB cAMP response element-binding protein

ECM Extracellular matrix

$\mathrm{HH}$ Hedgehog

IHH Indian Hedgehog

MAPK Mitogen-activated protein kinase

NFAT Nuclear factor of activated T cells

PAC1 Pituitary adenylate cyclase-activating polypeptide type I receptor

PACAP Pituitary adenylate cyclase polypeptide

PKA Protein kinase A

PKC Protein kinase $\mathrm{C}$

PP2A Protein phosphatase 2A

PP2B Protein phosphatase 2B

PTHrP Parathyroid hormone related peptide

Runx2 Runt-related transcription factor 2

SHH Sonic Hedgehog

TGF $\beta$ Transforming growth factor- $\beta$

VIP Vasoactive intestinal polypeptide

VPAC Vasoactive intestinal peptide receptor

Various signalling pathways influence the proper limb development and activating signals for many of them may arrive from the outer environment, partly via transmission by the surrounding extracellular matrix (ECM). Regulatory molecules or physical stimuli are able to induce the activation of specific receptors which can determine the cell fate during the differentiation cycle. Chemical signals reaching the tissue or directly the cells may originate from blood or interstitial fluid; in the latter case autocrine and paracrine signalling are the most frequent in developing organisms [1]. The best known mechanotransducer of developing cells is the primary cilium [2]. Changes of the intracellular ion concentration as the consequence of the activation of various ion channels can alter the resting membrane potential of cells and may influence proliferation and differentiation [3]. All of the processes mentioned above have impact on the cellular differentiation program of skeletal elements. Hyaline cartilage especially articular cartilage is an avascular and aneural tissue with a uniquely organized extracellular matrix. The sequential receptor activation by paracrine or autocrine ways plays crucial role in the differentiation of cartilage tissue, although many details of these mechanisms are still not well explored. The significance of the better understanding of cartilage formation is underscored by the fact, that production of proper long bone architecture requires a cartilage template and involves time and growth factor dependent activation of precisely defined regulating mechanisms and signalling cascade systems [4]. 


\section{PACAP and VIP}

VIP and PACAP are small hormone like peptides which belong to the VIP-secretingrowth hormone releasing hormone (GHRH)-glucagon superfamily. PACAP was first extracted from ovine hypothalamus, and was thought to be an important neurohormone regulating various processes in the CNS [5]. It is produced by a variety of cells and tissues in addition to neuronal cells. Among others, specific cells of the intestinal system can produce VIP along with some immune and endocrine cells. VIP has important functions in neuronal development and both in innate and acquired immunity [6].

PACAP has two bioactive forms: a shorter, 27 amino acid (PACAP 27) and a longer 38 amino acid (PACAP 38) variant [7]. The $\mathrm{N}$-terminal region of the polypeptide is evolutionary conserved and shows a high homology with that of VIP [5]. In the last decade, increasing amount of evidence has emerged regarding the important roles of PACAP in peripheral organs such as uterus [8], ovary [9], and teeth [10]; moreover, its presence has been proven in human milk [11]. Nonetheless, only sporadic data exist about its function in skeletal elements [12-15].

Three main $\mathrm{G}$ protein coupled receptors of these peptides have been identified such as PAC1, VPAC1, and VPAC2 from which PACAP binding to PAC1 has the highest affinity, while the latter two attract PACAP and VIP with equal affinity [16]. Alteration in the conformation of these receptors results in the elevation of intracellular cAMP level leading to the activation of protein kinase A (PKA) [5]. The so-called "canonical" signalling activation may lead to the nuclear translocation of CREB or Sox9 transcription factors and consequent activation of the expression of various genes. PAC1 receptor activation is also able to trigger the phosphorylation of key elements in MAPK pathways, such as ERK and p38 kinases [5], subsequently regulating cellular division or apoptotic program induction [17]. The versatility of PACAP/VIP receptor induced signal transduction mechanisms indicates its multifactorial regulation, implying a vast array of signalling connections. Activation of $\mathrm{IP}_{3}$ receptors inducing the release of $\mathrm{Ca}^{2+}$ from endoplasmic reticulum (ER) can be involved as the result of PACAP binding [18]. The elevation of intracellular $\mathrm{Ca}^{2+}$ concentration activates various $\mathrm{Ca}^{2+}$ dependent signalling molecules such as classical PKCs, MAPK [19] or protein phosphatases like PP2B [20]. G-protein coupled receptors (GPCR) have been proven to have communication with other signalling cascades, implying that PACAP receptor activation may cross talk with WNT $\beta$-catenin [21], TGF $\beta$ [22], BMP [23], Hedgehog [24], and Notch signal transduction [25]. Multifactorial or pleiotropic effects of PACAP have been investigated in several biological processes and it has been proven to prevent apoptosis, ischemic conditions, inflammation and oxidative stress [21, 26-29]. 


\section{PACAP and VIP Regulates Chondrogenic Differentiation}

The complete network of signalling cascades governing chondrogenic differentiation is not fully discovered. As articular cartilage has very poor regeneration capacity and no effective or curative treatment is available currently for degenerative cartilage diseases such as osteoarthritis the identification of new pharmacological targets for reconstruction of cartilage is very important. Hormone like peptides are not in the focus of research as blood vessels are not found in the articular cartilage and hormones can only be delivered by diffusion from the synovial fluid into the joint cartilage. Low level of nutrition and poor oxygenation also are consequences of the avascular nature of this tissue. Indeed, it can be a question of interest if the differentiating chondroprogenitor cells or chondrocytes are able to release small regulatory peptides and can influence their own differentiation via autocrine or paracrine ways.

Cartilage differentiation in vivo starts with rapid proliferation and aggregation of mesenchymal-like chondroprogenitor cells resulting in the formation of precartilage nodules, in which the final commitment of chondroprogenitor cells can be fulfilled. Chondrogenic nodules and cartilage specific extracellular matrix production are both required for proper hyaline cartilage structure formation [30]. The ECM of articular cartilage, composed mostly of high molecular mass proteoglycans (PG) such as aggrecan, glucosaminoglycans (GAG) like hyaluronan form a highly organized network attracting high amount of water. The extremely hydrated nature of the cartilage is essential to proper mechanical functions and offers substantial mechanical stability. Moreover, the negatively charged PGs and GAGs also function as an extracellular ion pool which can influence the normal lifecycle of chondrogenic cells [30]. Several transcription factors and receptors have been proven to play role in the matrix synthesis. One of these transcription factor families is the SoxE from which Sox5, Sox6, and Sox9 are essential for the induction of mRNA expression of cartilage matrix-specific proteins (e.g., COL2A1, aggrecan core protein). Sox 9 is one of the pivotal signalling elements of chondrogenesis and its regulation by reversible phosphorylation is a key momentum of the proper differentiation cycle [31]. Also CREB transcription factor regulates cartilage-specific matrix production directly and it plays an important role in lubricin secretion of articular cartilage [32]. Moreover, Sox 9 promoter is known to be regulated by the CREB that binds to a CRE site upstream of Sox 9 [33]. Our laboratory has demonstrated that Sox 9 and CREB transcription factors are phosphorylated by PKA during cartilage formation $[34,35]$. Nuclear translocation of the phosphorylated forms of these transcription factors enhances the matrix production of chondrocytes. Moreover, a complex regulatory mechanism and synergism between Sox9 function and the cAMP-PKACREB pathway including connections to the BMP signalling was published in both mature and differentiating chondrocytes $[34,36]$. We have shown that the activation of signalling elements phosphorylated by PKA can be equilibrated by Ser/Thr protein phosphatases such as PP2A and PP2B [37-39]. Our group also demonstrated that $\mathrm{PP} 2 \mathrm{~A}$ is a negative regulator of chondrogenesis and was involved in the 
mechanotransduction $[34,38]$. On the other hand, we showed that PP2B was a positive regulator of chondrogenesis and influenced the reversible phosphorylation of ERK1/2 and NFAT4 [37, 39]. In one of our previous works, we presented data that the changes in cytosolic free $\mathrm{Ca}^{2+}$ concentration showed a characteristic developmental stage dependent pattern in chondrogenic cells [40]. Additionally, differentiating stage dependent $\mathrm{Ca}^{2+}$ oscillations were identified in chondrogenic cells [41]. A wide range of receptors with substantial $\mathrm{Ca}^{2+}$ permeability have been described in the last decade as part of the mapping of chondrocyte specific channelome [3, 42].

Since the regulation of these signalling pathways which can modulate cartilage formation and chondrocyte biology is cAMP or $\mathrm{Ca}^{2+}$ dependent it implicates the importance of PACAP/VIP neuropeptides in proper hyaline cartilage formation. A potential role of VIP has been demonstrated in bone remodelling and it is known to have important functions in inflammatory diseases [43]. Moreover, this neuropeptide is able to regulate $\mathrm{Ca}^{2+}$ release of neonatal osteoblasts. VIP exerted a positive effect in rheumatoid arthritis indicating that its application in the therapy of this disorder can be promising [44]. Although the articular cartilage is aneural, the surrounding synovial membrane is rich in nerve endings, which release VIP into the synovial fluid and subsequently induce anti-inflammatory processes [45]. About the functions of PACAP in the adult joints we still have exiguous knowledge despite the fact that PACAP-positive nerve endings have been described in cartilage canals of porcine epiphyseal cartilage more than 15 years ago [46]. We have demonstrated that the mRNAs of preproPACAP as well as PAC1, VPAC1 and VPAC2 receptors are expressed in chicken "high density" chondrogenic cell cultures. Additionally, we have shown the expression of the PAC1 receptor protein in chondroprogenitor cells and an increased extracellular matrix synthesis was detected during PACAP administration suggesting the supportive effect of this neuropeptide in cartilage development [13]. Our findings implied the presence of PACAP-related autocrine and/or paracrine effects in cartilage itself, reflecting on a possible new signalling mechanism in the regeneration of hyaline cartilage $[47,48]$. In the light of these data Giunta and coworkers identified PACAP positive chondrocytes in the superficial zone of articular cartilage and in synovial fluid [12]. Moreover, the reduction of the neurohormone level was demonstrated during osteoarthritis in the articulating cartilage [12]. Although the expression of the VIP receptors has been detected in chondrogenic cells, there are some data pointing out that VIP probably does not influence matrix production of chondrocytes and synovial cells [49]. As classical downstream targets of PAC1 receptor, Gs and/or Gq proteins become activated triggering the elevation of intracellular cAMP concentration or the intracellular release of $\mathrm{Ca}^{2+}$ ions [5]. These events initiate the activation of certain protein kinases such as PKA, $\mathrm{PKC}$, and MAPK which all play essential, but probably distinct roles in chondrogenesis $[35,37,39]$. During proper chondrogenesis PKA is proved to phosphorylate CREB and Sox9 transcription factors $[31,35]$ by which induce the secretion of cartilage specific ECM components (Fig. 20.1). PACAP administration into the medium of chondrogenic cell cultures increased the phosphorylation both of Sox 9 and CREB, and enhanced matrix production of the differentiating cells by effecting on both the expression of aggrecan, collagen type II (Fig. 20.1) and the expression 


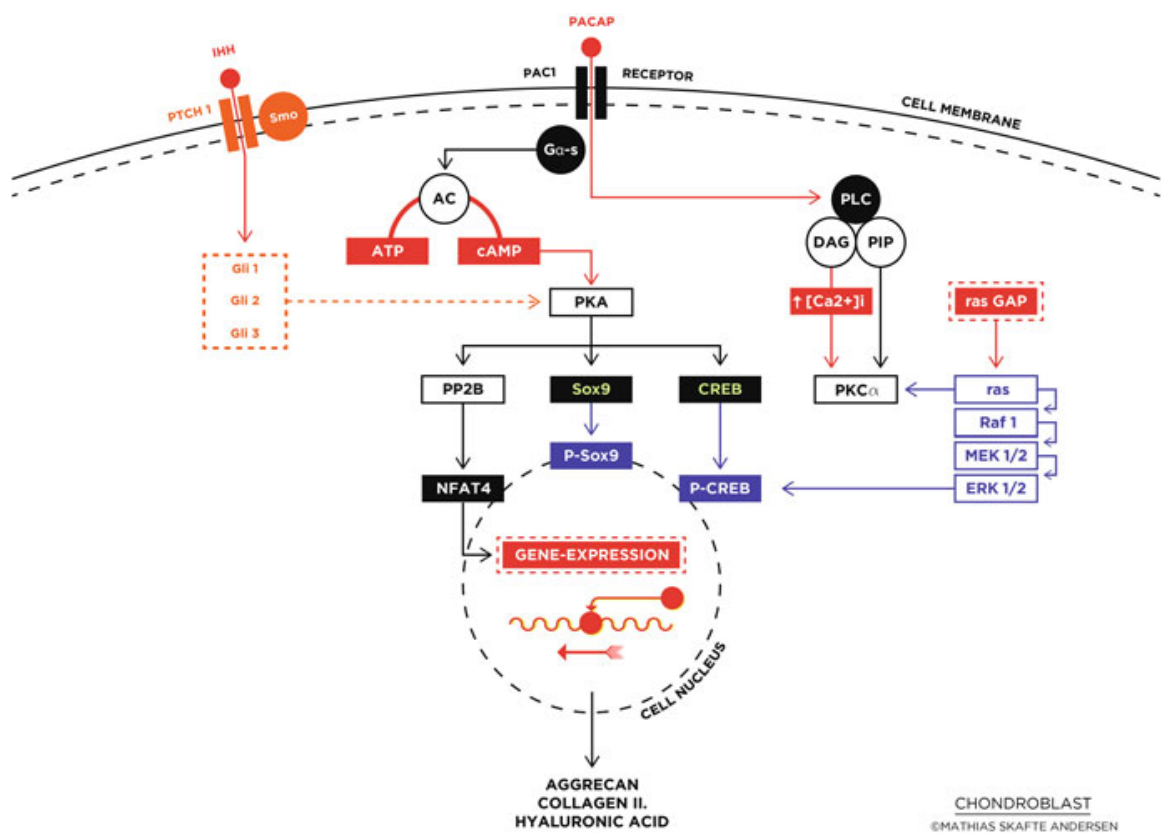

Fig. 20.1 Effects of PACAP on chondrogenic signalling mechanisms. The increased concentration of cAMP level elevates PKA activity. PKA phosphorylates CREB and Sox9 which translocate into the nucleus of chondrogenic cells and induce the gene expression of collagen type II, aggrecan, and various GAGs such as hyaluronic acid. Activation of PAC1 receptor can also elevate the intracellular $\mathrm{Ca}^{2+}$ concentration leading to increased PP2B, PKC, or MAPK signalling activity. The elevated activity of PP2B regulates NFAT4 being responsible for the augmented matrix production. PACAP has a connection with the IHH signalling pathway by decreasing the activity of Gli transcription factors

of matrix synthesizing enzymes such as Chst11, Extl1, or HAS enzymes [13]. PAC1 receptor activation also can be responsible for the elevation of intracellular $\mathrm{Ca}^{2+}$ concentration which in turn can activate the $\mathrm{Ca}^{2+}$ dependent phosphatase PP2B (also known as calcineurin). Therefore, we investigated the involvement of this Ser/Thr phosphatase in PACAP signalling pathways and connection between PP2B activity and PACAP signalling was proven [13] (Fig. 20.1). Similar observations were found in chromaffin cells [50]. These in vitro results indicate that the presence of PACAP is essential for proper cartilage formation although the phenotype of PACAP KO mice did not show any dramatic macroscopic morphological alteration of the skeleton [51]. Although the analysis of the genetically modified animals has not been completed yet, our initial observations suggested alterations in the composition of the cartilage extracellular matrix and in the expression of various signalling molecules in the knee joints of PACAP KO mice (our unpublished data). In the reproductory system of these mice, the lack of PACAP gene resulted in reduced fertility and altered mating behavior of females [52], moreover the maturation [53] and the morphology [54] of gonadal cells showed notable differences. Further evidences support the idea of the regulation effect of PACAP on differentiation of various cells, as 
alterations have been found in tooth formation of PACAP KO mice [10]. The complex phenotypic changes raise the possibility of multiple cross talk of PACAP signalling with developmental pathways connected to various morphogenes, as well as certain compensatory mechanisms of PACAP signalling cascades. For instance MAPK and Wnt signalling both play important roles in the proper cartilage formation and tissue patterning [55] and a PACAP-independent PAC1 receptor activation has been directly linked to the regulation of Wnt/ $\beta$-catenin pathways [21]. Notch signalling activation plays a crucial role in chondrogenesis [56] and exerts modulatory function in osteoarthritis [57]. Recently, a cross talk of G protein coupled receptors and Notch signalling has been reported in bacterial LPS induced macrophages [58]. SHH pathway is another essential positive chondroregulatory pathway [59] and it can be inhibited by PACAP activation [60].

Besides the direct influence of PACAP on chondrogenesis, we have demonstrated a chondro-protective effect of this neuropeptide in chondrogenic cell cultures where its administration compensated the harmful effects of oxidative stress. Similar phenomenon has been observed in ischemia or oxidative stress induced apoptosis in the central nervous system [61] or in diabetic kidney [62]. Moreover, PACAP deficient mice showed higher sensitivity to injury during retinal ischemic conditions, axonal lesion, intestinal inflammation or oxidative stress of the kidneys [63]. The activation of PACAP/VIP system had positive effect in rheumatoid arthritis $[64,65]$ and decreased expression of PACAP was identified in osteoarthritic knee joint [12]. PACAP is known to exert cytoprotective effects in several other peripheral organs and in the CNS, for example cardioprotective effects of these peptides have been demonstrated [66] and a positive effect in Parkinson's disease also have been shown [67]. On the basis of these observations, a protective and/or ameliorating effect of PACAP was likely in diseases of the articulating cartilage or skeletal stress situations. Indeed, the addition of PACAP1-38 during oxidative stress prevented the inhibition of cartilage matrix production by normalizing the phosphorylation of Sox9 and CREB in chicken chondrogenic cells [13]. Recently, the involvement of PACAP or VIP signalling activation in mechanotransduction of developing articular cartilage has been proved by our group [68] and the importance of PKA in mechanical cellular response was proposed [34]. Mechanical load of in vitro chondrogenic cell cultures resulted in an increased PAC1 receptor and PACAP expression, and supported the undifferentiated stage of chondroblasts. Mechanical load of in vitro chondrogenic cell cultures resulted in increased PAC1 receptor and PACAP expression and the activation of IHH (Fig. 20.1) inducing the elevated expression of collagen type $\mathrm{X}$ which was normalized by PACAP 1-38 addition [68]. In these experiments, we found that PACAP administration was able to reduce the expression of matrix metalloproteinases (MMP) during oxidative stress in chondrogenic cell cultures (our unpublished data). Similar results have been published in alveolar cells where both VIP and PACAP were able to decrease the expression of certain MMPs and reduced the activation and expression of caspase3 [69]. It is also important to note that VIP and its receptors in synovial fibroblasts [70] are able to regulate inflammatory factors release [71]. These data all strongly suggest that PACAP is a promising future therapeutic agent in inflammatory and degenerative joint diseases [72]. 


\section{Bone Formation Under the Control of VIP and PACAP Signalling Cascades}

Development of long bones is determined and organized by a cartilage template. Invasion of the calcified cartilage by the osteoprogenitor cells and their precisely regulated differentiation are required for the proper osteogenesis [4]. Development of bone tissue is supported by complex bone specific developing mechanisms and signalling. Differentiation of osteoblasts from osteoprogenitors is followed by an initial deposition of a bone specific organic ECM abundant in collagen type I completed with another bone specific matrix components such as osteocalcin or osteonectin [4]. Calcification of bone matrix also requires osteoblast activity, calcium hydroxyapatite crystals accumulate and deposit into the collagen rich bone matrix. Various signalling cascades are considered as bone specific regulatory mechanisms, such as BMP, WNT, Notch, and Hedgehog signalling pathways [73-77]. Thyroid hormones are crucial components of bone remodelling and differentiation and neuronal connections are also found to be involved in bone production [74]. Consequently, the activation of signalling mechanisms mentioned above by hormones and neuropeptides may derive from different sources in the surrounding tissues. The presence and precise timing of the expression of growth factors such as BMPs are essential for proper bone production. These factors bind to their own receptors; to BMPRs. Subsequently, their activation induces the phosphorylation of Smad1/5 and with the help of Smad4 the complex is translocated into the nuclei of osteogenic cells and initiates expression of bone specific genes (Fig. 20.2.) such as the transcription factor osterix, alkaline phosphatase (ALP), or collagen type I [78, 79]. Sequential release of BMPs is regulated by complex networks of signalling cascades including CREB, one of the transcription factors activated via PKA signalling pathways [79] and activate the gene expression of the proteins mentioned previously (Fig. 20.2). On the other hand a well-balanced expression of hedgehog signalling elements governed by another bone specific transcription factor, Runx 2 is also essential for proper long bone formation [80] (Fig. 20.2). Runx 2 can be directly phosphorylated by PKA [81] and subsequently activates the expression of bone specific signalling elements or ECM components (Fig. 20.2). FGFs are also essential for proper bone formation. FGF2 decreases the ALP activity, Runx2 activation, and collagen secretion and elevates the proliferation of osteoblasts [82]. This complex signalization involves broad spectrum cross talk opportunities with the PACAP/ VIP signalization, further highlighting the significance of neuropeptide signalling in bone formation and regeneration.

At the beginning of enchondral ossification, blood vessels invade into the bone template, in a process regulated by VEGFs. The expression of these growth factors is also under the control of PKA-Runx2 axis [83]. Formation and invasion of the blood vessels into the calcified cartilage matrix result in the appearance of osteoprogenitor cells and differentiation of osteoblasts producing the bone matrix. This process can also be regulated by neuropeptides [84] or invading neuronal elements. During the elongation of long bones PACAP positive nerve fibers have been shown 


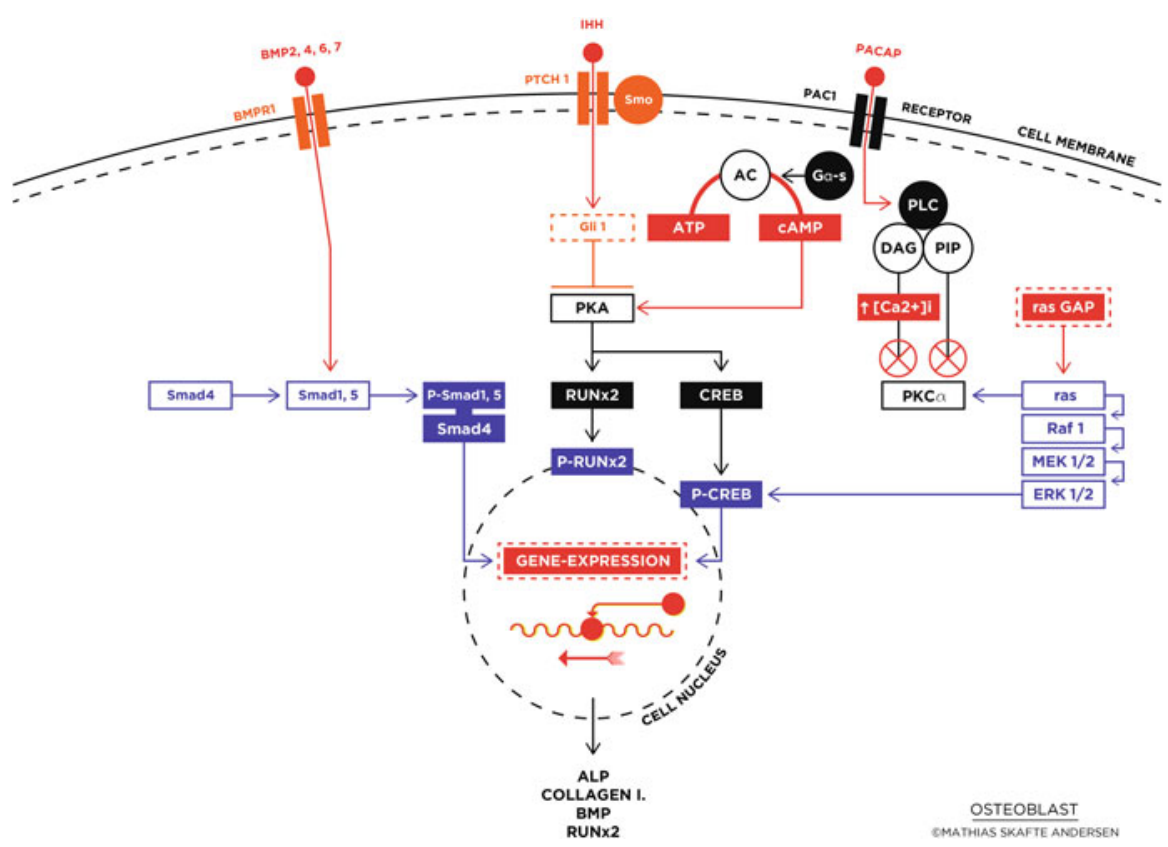

Fig. 20.2 Diverse connections of PACAP signalling pathways in osteogenic cells. PACAP binding to its receptors elevates the intracellular cAMP concentration and activates PKA in osteoblast cells. CREB is partly phosphorylated by PKA while the nuclear translocation of Runx 2 is regulated by the kinase. The presence of PACAP does not induce intracellular $\mathrm{Ca}^{2+}$ release, subsequently the $\mathrm{Ca}^{2+}$ dependent signalling pathways are not activated (arrows crossed by red lines). The neuropeptide trigger the expression of BMPs simultaneously activated Smad transcription factors and cross talk with Runx2. SHH or IHH binding to PTCH1 receptor can induce the nuclear translocation of Gli1 transcription factor. Activation of Gli1 is inhibited by the continuous activation of PKA

penetrating the bone matrix [46], and VIP positive sympathetic nerve endings have also been identified releasing these neuropeptides [85]. As an interesting observation, receptor composition and effects of VIP exhibited differences in cells of bones developed in different ways, i.e., via membranous or endochondral ossification. Moreover, the direct communication of sympathetic nerve fibers with osteoblasts showed an embryonic origin dependent response and signalization, suggesting that the innervation of periosteum by peptidergic fibers plays important function both in bone regeneration and formation [86].

It has been proven that in calvaria derived cell lines, in which the receptors and proteins of PACAP and/or VIP signalling are present, PKA activation can be induced by PACAP or VIP addition [14, 87]. Accumulation of cAMP in osteoblasts is proved to be a result of combined activation of PACAP/VIP and regulates diverse signalling pathways influencing osteoblast differentiation. In line with this, presence of certain neuropeptides was shown to be elevated after bone fracture, indicating their importance in successful regeneration [88]. A recent report demonstrated release of various 
neuropeptides from periosteal nerve endings resulting in enhancement of intercellular communication and increased metabolic activity of osteoblasts [89].

As it was described above, osteogenic transformation, bone matrix production and mineralization are regulated by multiple signalling cascades [90], where the activation of MAPK and PKA plays essential roles. Runx2 is one of the key transcription factors and it governs osteoblast differentiation [91] and it is regulated by PKA signalling pathways [92].

Our laboratory has proven that the administration of PACAP into the medium of UMR-106 osteoblast cell line enhanced the nuclear translocation of Runx2 and an increased expression of collagen type I, ALP and osterix genes was observed (Fig. 20.2). Interestingly, the phosphorylation level of CREB transcription factors was not significantly increased [14], raising the possibility of the participation of MAPK signalling cascade as an alternative signalling pathway for the Runx2 regulation [93].

BMP signalling pathway is another fundamental regulator of osteogenesis and cross talk with Runx2 has been reported [94] (Fig. 20.2). Moreover, the TGF $\beta /$ BMP pathways are activated by PACAP or VIP [23]. Indeed, the expression of BMP6 and 7 elevated in the presence of PACAP in UMR-106 cells indicating a strong positive effect of the neuropeptide in bone differentiation. Moreover, as a sign of BMPR activation, a pronounced elevation of the nuclear presence of Smad1 transcription factor was detected under the effect of PACAP administration [14]. TGF $\beta /$ BMP signalling pathways are involved in the regulation and activation of VIP signalling cascades. Exogenous VIP may also result in the activation of Smads [95] suggesting a complex signalling with numerous alternative routes during bone development [14].

The involvement of MAPK system has been reported in bone development and in fracture healing. The administration of PACAP and VIP is proven to upregulate the activation of ERK $1 / 2$ in osteoblasts [96, 97] or adipogenic models [98]. The two neurohormones have negative effect on JNK and p38 phosphorylation in monocytes in vivo and in vitro [99]. Additionally, intracellular $\mathrm{Ca}^{2+}$ concentration can be elevated by PACAP [100] or VIP [101], resulting in an activation of classical PKCs and ERK both influencing osteoblast differentiation [102]. Nonetheless, in UMR106 cell line we were not able to detect significant $\mathrm{Ca}^{2+}$ concentration change and no significant alteration was detected in activation of classical PKCs such as PKC $\alpha$ [14] (Fig. 20.2). $\mathrm{Ca}^{2+}$ influx can be evoked by PACAP [101] PACAP and VIP are able to decrease the $\mathrm{Ca}^{2+}$ entry via L- and N-type calcium channels in neurons [102]. It is known that the administration of PACAP affects $\mathrm{Ca}^{2+}$ oscillation [103] and alters the $\mathrm{Ca}^{2+}$ related vesicular transport of chromaffin cells [104]. Besides this dynamic alteration of intracellular Ca-homeostasis, PACAP also exerts effects on matrix mineralization. We have reported that the inorganic matrix components of UMR-106 cell line can be elevated by PACAP addition [14]. Moreover, an altered mineralization was detected during tooth formation of PACAP deficient mice [10], suggesting a yet unknown connection between PACAP and mineralized tissue formation. As a possible mechanism for PACAP induced extracellular $\mathrm{Ca}^{2+}$ accumulation during osteogenesis, calcitonin gene-related peptide was proven to influence 
osteoclast function [105] and the presence of PACAP decreased the matrix-resorption and consequent Ca-release by these cells [90, 106].

Hedgehog signalling is of key importance amongst the regulatory mechanisms of bone and cartilage development [80]. A well-defined balance between Indian hedgehog (IHH) and parathyroid hormone related peptide (PTHrP) is essential for proper long bone formation, regulation of proliferation, and matrix production of osteoblasts via the activation of Runx2 transcription factor [107]. PTHrP directly communicates with PKA signalling inducing the activation of CREB and NFAT factors in osteoblasts [108]. In UMR-106 cells the application of PACAP elevated the expression of PTHrP without altering the IHH expression [14]. Sonic hedgehog (SHH) pathway is known to be regulated by PACAP signalling [60] and the activation of PKA downregulates the function of Gli1, which consequently decreases the proliferation [24] (Fig. 20.2). In PACAP KO mice, enhanced SHH signalling was detected during tooth development [10]. On the contrary, exogenous administration of PACAP elevated the expression of SHH and a more pronounced nuclear presence of Gli1 was found in rat UMR-106 cells [14]. This contradiction may stem from the osteosarcoma origin of UMR cells, as malignant cells can exhibit alterations of various signalling mechanisms. Another hypothesis is that the cellular presence of the repressor form of Gli2 or Gli3 transcription factors are also increased in UMR106 cells which upregulate the activation of SHH signalling pathways in this tumor cell line. Nevertheless, we have only sparse data about the function of VIP in bone development, but it is a suppressor of bone resorption and has very similar effect as mechanical load in MC3T3 osteoblastic cell line [43].

\section{Conclusion}

Articular cartilage is optimally able to dissipate the mechanical stress, which loads the articular surface and transmits it to the subchondral bone. Chondrocytes represent the only type of cells in cartilage with a very limited capacity to reproduce themselves even in a healthy adult human. Therefore, they cannot regenerate the cartilage tissue in case of traumatic injury or loss of joint surface, which can be the ultimate consequence of any type of inflammatory joint diseases. Protection of this tissue during joint inflammation, stimulation of its poor regeneration capacity or production and implantation of artificial cartilage all are major challenges of the modern reparative medicine. PACAP was originally described as a hormone-like product of neurons, able to reduce the harmful consequences of various brain injuries, as well as playing regulatory roles during brain development. On the basis of the data presented and summarized above, PACAP and VIP are important modulators of the physiological differentiating processes of articular cartilage and may influence endochondral ossification. As PACAP and VIP neuropeptides are naturally synthetized signal molecules of humans, they can be good candidates for application via intraarticular injection as chondroprotective agents or they can be interesting candidates for the regulation of callus formation. 
Acknowledgements The authors are grateful to Mrs. Krisztina Bíró for excellent technical assistance and to Ms. Andrea Pálfi, Ms. Solveig Lind Helgadottir, Ms. Katarina Karadottir, and Mr. Vince Szegecki medical students for their skillful help during the studies and for Mathias Skafte Andersen for the graphical figures. This work was supported by grants from Akira Arimura Foundation Research Grant, the Hungarian Science Research Fund (OTKA 119759 and OTKA K 104984), PTE AOK KA Research Grant, Bolyai Scholarship, and the Hungarian Academy of Sciences (MTA "Lendület" Program) and from the New Széchenyi Plan (TÁMOP-4.2.2.A-11/1/ KONV-2012-0053; The project is co-financed by the European Union and the European Social Fund). This research and T.J. was supported by Szodoray Lajos and Magyary Zoltán Funds by the European Union and the State of Hungary, co-financed by the European Social Fund in the framework of TÁMOP 4.2.4. A/2-11-1-2012-0001 "National Excellence Program" New National Excellence Program (UNKP). This work is dedicated to the 650th anniversary of the University of Pecs. T.J. and R.Z. are supported by GOP-1.1.1-11-2012-0197 financed by the Hungarian government and the EU. This work was supported by the University of Debrecen (RH/751/2015).

\section{References}

1. Yan JY, Tian FM, Wang WY, Cheng Y, Song HP, Zhang YZ, et al. Parathyroid hormone (1-34) prevents cartilage degradation and preserves subchondral bone micro-architecture in guinea pigs with spontaneous osteoarthritis. Osteoarthritis Cartilage. 2014;22:1869-77.

2. Rais Y, Reich A, Simsa-Maziel S, Moshe M, Idelevich A, Kfir T, et al. The growth plate's response to load is partially mediated by mechano-sensing via the chondrocytic primary cilium. Cell Mol Life Sci. 2015;72:597-615.

3. Barrett-Jolley R, Lewis R, Fallman R, Mobasheri A. The emerging chondrocyte channelome. Front Physiol. 2010;1:135.

4. Paiva KB, Granjeiro JM. Bone tissue remodeling and development: focus on matrix metalloproteinase functions. Arch Biochem Biophys. 2014;561C:74-87.

5. Vaudry D, Falluel-Morel A, Bourgault S, Basille M, Burel D, Wurtz O, et al. Pituitary adenylate cyclase-activating polypeptide and its receptors: 20 years after the discovery. Pharmacol Rev. 2009;61:283-357.

6. Moody TW, Hill JM, Jensen RT. VIP as a trophic factor in the CNS and cancer cells. Peptides. 2003;24:163-77.

7. Miyata A, Arimura A, Dahl RR, Minamino N, Uehara A, Jiang L, et al. Isolation of a novel 38 residue-hypothalamic polypeptide which stimulates adenylate cyclase in pituitary cells. Biochem Biophys Res Commun. 1989;164:567-74.

8. Reglodi D, Tamas A, Koppan M, Szogyi D, Welke L. Role of PACAP in female fertility and reproduction at gonadal level-recent advances. Front Endocrinol (Lausanne). 2012;3:155.

9. Koppan M, Varnagy A, Reglodi D, Brubel R, Nemeth J, Tamas A, et al. Correlation between oocyte number and follicular fluid concentration of pituitary adenylate cyclase-activating polypeptide (PACAP) in women after superovulation treatment. J Mol Neurosci. 2012;48:617-22.

10. Sandor B, Fintor K, Felszeghy S, Juhasz T, Reglodi D, Mark L, et al. Structural and morphometric comparison of the molar teeth in pre-eruptive developmental stage of PACAP-deficient and wild-type mice. J Mol Neurosci. 2014;54:331-41.

11. Csanaky K, Banki E, Szabadfi K, Reglodi D, Tarcai I, Czegledi L, et al. Changes in PACAP immunoreactivity in human milk and presence of PAC1 receptor in mammary gland during lactation. J Mol Neurosci. 2012;48:631-7.

12. Giunta S, Castorina A, Marzagalli R, Szychlinska MA, Pichler K, Mobasheri A, et al. Ameliorative effects of PACAP against cartilage degeneration. Morphological, immunohistochemical and biochemical evidence from in vivo and in vitro models of rat osteoarthritis. Int J Mol Sci. 2015;16:5922-44. 
13. Juhasz T, Matta C, Katona E, Somogyi C, Takacs R, Gergely P, et al. Pituitary adenylate cyclase activating polypeptide (PACAP) signalling exerts chondrogenesis promoting and protecting effects: implication of calcineurin as a downstream target. PLoS One. 2014;9:e91541.

14. Juhasz T, Matta C, Katona E, Somogyi C, Takacs R, Hajdu T, et al. Pituitary adenylate cyclase-activating polypeptide (PACAP) signalling enhances osteogenesis in UMR-106 cell line. J Mol Neurosci. 2014;54:555-73.

15. Kovacs CS, Chik CL, Li B, Karpinski E, Ho AK. Pituitary adenylate cyclase-activating peptide stimulates cyclic AMP accumulation in UMR 106 osteoblast-like cells. J Endocrinol. 1996;149:287-95.

16. Gourlet P, Vandermeers A, Vertongen P, Rathe J, De NP, Cnudde J, et al. Development of high affinity selective VIP1 receptor agonists. Peptides. 1997;18:1539-45.

17. Dohi K, Mizushima H, Nakajo S, Ohtaki H, Matsunaga S, Aruga T, et al. Pituitary adenylate cyclase-activating polypeptide (PACAP) prevents hippocampal neurons from apoptosis by inhibiting JNK/SAPK and p38 signal transduction pathways. Regul Pept. 2002;109:83-8.

18. Tanaka K, Shibuya I, Uezono Y, Ueta Y, Toyohira Y, Yanagihara N, et al. Pituitary adenylate cyclase-activating polypeptide causes $\mathrm{Ca} 2+$ release from ryanodine/caffeine stores through a novel pathway independent of both inositol trisphosphates and cyclic AMP in bovine adrenal medullary cells. J Neurochem. 1998;70:1652-61.

19. Szabo A, Danyadi B, Bognar E, Szabadfi K, Fabian E, Kiss P, et al. Effect of PACAP on MAP kinases, Akt and cytokine expressions in rat retinal hypoperfusion. Neurosci Lett. 2012;523:93-8.

20. Schuhmann K, Romanin C, Baumgartner W, Groschner K. Intracellular Ca2+ inhibits smooth muscle $\mathrm{L}$-type $\mathrm{Ca} 2+$ channels by activation of protein phosphatase type $2 \mathrm{~B}$ and by direct interaction with the channel. J Gen Physiol. 1997;110:503-13.

21. Yu R, Cui Z, Li M, Yang Y, Zhong J. Dimer-dependent intrinsic/basal activity of the class B G protein-coupled receptor PAC1 promotes cellular anti-apoptotic activity through Wnt/ beta-catenin pathways that are associated with dimer endocytosis. PLoS One. 2014;9:e113913.

22. Oka H, Jin L, Kulig E, Scheithauer BW, Lloyd RV. Pituitary adenylate cyclase-activating polypeptide inhibits transforming growth factor-beta1-induced apoptosis in a human pituitary adenoma cell line. Am J Pathol. 1999;155:1893-900.

23. Pavelock KA, Girard BM, Schutz KC, Braas KM, May V. Bone morphogenetic protein down-regulation of neuronal pituitary adenylate cyclase-activating polypeptide and reciprocal effects on vasoactive intestinal peptide expression. J Neurochem. 2007;100:603-16.

24. Niewiadomski P, Zhujiang A, Youssef M, Waschek JA. Interaction of PACAP with Sonic hedgehog reveals complex regulation of the hedgehog pathway by PKA. Cell Signal. 2013;25:2222-30.

25. Lu Q, Tong B, Luo Y, Sha L, Chou G, Wang Z, et al. Norisoboldine suppresses VEGFinduced endothelial cell migration via the cAMP-PKA-NF-kappaB/Notch1 pathway. PLoS One. 2013;8:e81220.

26. Botz B, Bolcskei K, Kereskai L, Kovacs M, Nemeth T, Szigeti K, et al. Differential regulatory role of pituitary adenylate cyclase-activating polypeptide in the serum-transfer arthritis model. Arthritis Rheumatol. 2014;66:2739-50.

27. Fabian E, Reglodi D, Mester L, Szabo A, Szabadfi K, Tamas A, et al. Effects of PACAP on intracellular signaling pathways in human retinal pigment epithelial cells exposed to oxidative stress. J Mol Neurosci. 2012;48:493-500.

28. Heimesaat MM, Dunay IR, Schulze S, Fischer A, Grundmann U, Alutis M, et al. Pituitary adenylate cyclase-activating polypeptide ameliorates experimental acute ileitis and extraintestinal sequelae. PLoS One. 2014;9:e108389.

29. Rozzi SJ, Borelli G, Ryan K, Steiner JP, Reglodi D, Mocchetti I, et al. PACAP27 is protective against tat-induced neurotoxicity. J Mol Neurosci. 2014;54:485-93.

30. Goldring MB, Tsuchimochi K, Ijiri K. The control of chondrogenesis. J Cell Biochem. 2006;97:33-44. 
31. de Crombrugghe B, Lefebvre V, Behringer RR, Bi W, Murakami S, Huang W. Transcriptional mechanisms of chondrocyte differentiation. Matrix Biol. 2000;19:389-94.

32. Ogawa H, Kozhemyakina E, Hung HH, Grodzinsky AJ, Lassar AB. Mechanical motion promotes expression of Prg4 in articular cartilage via multiple CREB-dependent, fluid flow shear stress-induced signaling pathways. Genes Dev. 2014;28:127-39.

33. Piera-Velazquez S, Hawkins DF, Whitecavage MK, Colter DC, Stokes DG, Jimenez SA. Regulation of the human SOX9 promoter by Sp1 and CREB. Exp Cell Res. 2007;313:1069-79.

34. Juhasz T, Matta C, Somogyi C, Katona E, Takacs R, Soha RF, et al. Mechanical loading stimulates chondrogenesis via the PKA/CREB-Sox9 and PP2A pathways in chicken micromass cultures. Cell Signal. 2014;26:468-82.

35. Zakany R, Szucs K, Bako E, Felszeghy S, Czifra G, Biro T, et al. Protein phosphatase 2A is involved in the regulation of protein kinase A signaling pathway during in vitro chondrogenesis. Exp Cell Res. 2002;275:1-8.

36. Zhao L, Li G, Zhou GQ. SOX9 directly binds CREB as a novel synergism with the PKA pathway in BMP-2-induced osteochondrogenic differentiation. J Bone Miner Res. 2009;24:826-36.

37. Juhasz T, Matta C, Mészár Z, Nagy G, Szijgyarto Z, Molnar Z, et al. Optimalized transient transfection of chondrogenic primary cell cultures. CEJB. 2010;5:572-84.

38. Zakany R, Bako E, Felszeghy S, Hollo K, Balazs M, Bardos H, et al. Okadaic acid-induced inhibition of protein phosphatase $2 \mathrm{~A}$ enhances chondrogenesis in chicken limb bud micromass cell cultures. Anat Embryol (Berl). 2001;203:23-34.

39. Zakany R, Szijgyarto Z, Matta C, Juhasz T, Csortos C, Szucs K, et al. Hydrogen peroxide inhibits formation of cartilage in chicken micromass cultures and decreases the activity of calcineurin: implication of ERK1/2 and Sox9 pathways. Exp Cell Res. 2005;305:190-9.

40. Matta C, Fodor J, Szijgyarto Z, Juhasz T, Gergely P, Csernoch L, et al. Cytosolic free Ca2+ concentration exhibits a characteristic temporal pattern during in vitro cartilage differentiation: a possible regulatory role of calcineurin in Ca-signalling of chondrogenic cells. Cell Calcium. 2008;44:310-23.

41. Fodor J, Matta C, Olah T, Juhasz T, Takacs R, Toth A, et al. Store-operated calcium entry and calcium influx via voltage-operated calcium channels regulate intracellular calcium oscillations in chondrogenic cells. Cell Calcium. 2013;54:1-16.

42. Somogyi CS, Matta C, Foldvari Z, Juhasz T, Katona E, Takacs AR, et al. Polymodal transient receptor potential vanilloid (TRPV) ion channels in chondrogenic cells. Int J Mol Sci. 2015;16:18412-38.

43. Yoo YM, Kwag JH, Kim KH, Kim CH. Effects of neuropeptides and mechanical loading on bone cell resorption in vitro. Int J Mol Sci. 2014;15:5874-83.

44. Sethi V, Rubinstein I, Kuzmis A, Kastrissios H, Artwohl J, Onyuksel H. Novel, biocompatible, and disease modifying VIP nanomedicine for rheumatoid arthritis. Mol Pharm. 2013;10:728-38.

45. Konttinen YT, Tiainen VM, Gomez-Barrena E, Hukkanen M, Salo J. Innervation of the joint and role of neuropeptides. Ann N Y Acad Sci. 2006;1069:149-54.

46. Strange-Vognsen HH, Arnbjerg J, Hannibal J. Immunocytochemical demonstration of pituitary adenylate cyclase activating polypeptide (PACAP) in the porcine epiphyseal cartilage canals. Neuropeptides. 1997;31:137-41.

47. Ahmed N, Dreier R, Gopferich A, Grifka J, Grassel S. Soluble signalling factors derived from differentiated cartilage tissue affect chondrogenic differentiation of rat adult marrow stromal cells. Cell Physiol Biochem. 2007;20:665-78.

48. Gelse K, Brem M, Klinger P, Hess A, Swoboda B, Hennig F, et al. Paracrine effect of transplanted rib chondrocyte spheroids supports formation of secondary cartilage repair tissue. J Orthop Res. 2009;27:1216-25.

49. Rahman S, Dobson PR, Bunning RA, Russell RG, Brown BL. The regulation of connective tissue metabolism by vasoactive intestinal polypeptide. Regul Pept. 1992;37:111-21. 
50. Lee HW, Hahm SH, Hsu CM, Eiden LE. Pituitary adenylate cyclase-activating polypeptide regulation of vasoactive intestinal polypeptide transcription requires $\mathrm{Ca} 2+$ influx and activation of the serine/threonine phosphatase calcineurin. J Neurochem. 1999;73:1769-72.

51. Hattori S, Takao K, Tanda K, Toyama K, Shintani N, Baba A, et al. Comprehensive behavioral analysis of pituitary adenylate cyclase-activating polypeptide (PACAP) knockout mice. Front Behav Neurosci. 2012;6:58.

52. Shintani N, Mori W, Hashimoto H, Imai M, Tanaka K, Tomimoto S, et al. Defects in reproductive functions in PACAP-deficient female mice. Regul Pept. 2002;109:45-8.

53. Barberi M, Di Paolo V, Latini S, Guglielmo MC, Cecconi S, Canipari R. Expression and functional activity of PACAP and its receptors on cumulus cells: effects on oocyte maturation. Mol Cell Endocrinol. 2013;375:79-88.

54. Brubel R, Kiss P, Vincze A, Varga A, Varnagy A, Bodis J, et al. Effects of pituitary adenylate cyclase activating polypeptide on human sperm motility. J Mol Neurosci. 2012;48:623-30.

55. Zhang Y, Pizzute T, Pei M. A review of crosstalk between MAPK and Wnt signals and its impact on cartilage regeneration. Cell Tissue Res. 2014;358:633-49.

56. Serrano MJ, So S, Hinton RJ. Roles of notch signalling in mandibular condylar cartilage. Arch Oral Biol. 2014;59:735-40.

57. Sassi N, Gadgadi N, Laadhar L, Allouche M, Mourali S, Zandieh-Doulabi B, et al. Notch signaling is involved in human articular chondrocytes de-differentiation during osteoarthritis. J Recept Signal Transduct Res. 2014;34:48-57.

58. Sangphech N, Osborne BA, Palaga T. Notch signaling regulates the phosphorylation of Akt and survival of lipopolysaccharide-activated macrophages via regulator of $\mathrm{G}$ protein signaling 19 (RGS19). Immunobiology. 2014;219:653-60.

59. Kwon HJ. ATP oscillations mediate inductive action of FGF and Shh signalling on prechondrogenic condensation. Cell Biochem Funct. 2013;31:75-81.

60. Waschek JA, Cicco-Bloom E, Nicot A, Lelievre V. Hedgehog signaling: new targets for GPCRs coupled to cAMP and protein kinase A. Ann N Y Acad Sci. 2006;1070:120-8.

61. Sanchez A, Chiriva-Internati M, Grammas P. Transduction of PACAP38 protects primary cortical neurons from neurotoxic injury. Neurosci Lett. 2008;448:52-5.

62. Banki E, Kovacs K, Nagy D, Juhasz T, Degrell P, Csanaky K, et al. Molecular mechanisms underlying the nephroprotective effects of PACAP in diabetes. J Mol Neurosci. 2014;54:300-9.

63. Reglodi D, Kiss P, Szabadfi K, Atlasz T, Gabriel R, Horvath G, et al. PACAP is an endogenous protective factor-insights from PACAP-deficient mice. J Mol Neurosci. 2012;48:482-92.

64. Hernanz A, Medina S, de Miquel E, Martin-Mola E. Effect of calcitonin gene-related peptide, neuropeptide $\mathrm{Y}$, substance $\mathrm{P}$, and vasoactive intestinal peptide on interleukin-1beta, interleukin- 6 and tumor necrosis factor-alpha production by peripheral whole blood cells from rheumatoid arthritis and osteoarthritis patients. Regul Pept. 2003;115:19-24.

65. Pulsatelli L, Dolzani P, Silvestri T, De GR, Salvarani C, Macchioni P, et al. Synovial expression of vasoactive intestinal peptide in polymyalgia rheumatica. Clin Exp Rheumatol. 2006;24:562-6.

66. Dvorakova MC. Cardioprotective role of the VIP signaling system. Drug News Perspect. 2005;18:387-91.

67. Reglodi D, Kiss P, Lubics A, Tamas A. Review on the protective effects of PACAP in models of neurodegenerative diseases in vitro and in vivo. Curr Pharm Des. 2011;17:962-72.

68. Juhasz T, Szentleleky E, Somogyi CS, Takacs R, Dobrosi N, Engler M, et al. Pituitary adenylate cyclase activating polypeptide (PACAP) pathway is induced by mechanical load and reduces the activity of hedgehog signaling in chondrogenic micromass cell cultures. Int J Mol Sci. 2015; 16:17344-67.

69. Onoue S, Ohmori Y, Endo K, Yamada S, Kimura R, Yajima T. Vasoactive intestinal peptide and pituitary adenylate cyclase-activating polypeptide attenuate the cigarette smoke extractinduced apoptotic death of rat alveolar L2 cells. Eur J Biochem. 2004;271:1757-67. 
70. Juarranz Y, Gutierrez-Canas I, Santiago B, Carrion M, Pablos JL, Gomariz RP. Differential expression of vasoactive intestinal peptide and its functional receptors in human osteoarthritic and rheumatoid synovial fibroblasts. Arthritis Rheum. 2008;58:1086-95.

71. Carrion M, Perez-Garcia S, Jimeno R, Juarranz Y, Gonzalez-Alvaro I, Pablos JL, et al. Inflammatory mediators alter interleukin-17 receptor, interleukin-12 and -23 expression in human osteoarthritic and rheumatoid arthritis synovial fibroblasts: immunomodulation by vasoactive intestinal peptide. Neuroimmunomodulation. 2013;20:274-84.

72. Mobasheri A. The future of osteoarthritis therapeutics: emerging biological therapy. Curr Rheumatol Rep. 2013;15:385.

73. Chen G, Deng C, Li YP. TGF-beta and BMP signaling in osteoblast differentiation and bone formation. Int J Biol Sci. 2012;8:272-88.

74. Gogakos AI, Duncan Bassett JH, Williams GR. Thyroid and bone. Arch Biochem Biophys. 2010;503:129-36.

75. Kim JH, Liu X, Wang J, Chen X, Zhang H, Kim SH, et al. Wnt signaling in bone formation and its therapeutic potential for bone diseases. Ther Adv Musculoskelet Dis. 2013;5:13-31.

76. Pan A, Chang L, Nguyen A, James AW. A review of hedgehog signaling in cranial bone development. Front Physiol. 2013;4:61.

77. Yavropoulou MP, Yovos JG. The role of Notch signaling in bone development and disease. Hormones (Athens). 2014;13:24-37.

78. Wang L, Park P, La MF, Than K, Rahman S, Lin CY. Bone formation induced by BMP-2 in human osteosarcoma cells. Int J Oncol. 2013;43:1095-102.

79. Zhang R, Edwards JR, Ko SY, Dong S, Liu H, Oyajobi BO, et al. Transcriptional regulation of BMP2 expression by the PTH-CREB signaling pathway in osteoblasts. PLoS One. 2011;6:e20780.

80. Ehlen HW, Buelens LA, Vortkamp A. Hedgehog signaling in skeletal development. Birth Defects Res C Embryo Today. 2006;78:267-79.

81. Jonason JH, Xiao G, Zhang M, Xing L, Chen D. Post-translational regulation of Runx2 in bone and cartilage. J Dent Res. 2009;88:693-703.

82. Hughes-Fulford M, Li CF. The role of FGF-2 and BMP-2 in regulation of gene induction, cell proliferation and mineralization. J Orthop Surg Res. 2011;6:8.

83. Yang J, Zhao L, Tian W, Liao Z, Zheng H, Wang G, et al. Correlation of WWOX, RUNX2 and VEGFA protein expression in human osteosarcoma. BMC Med Genomics. 2013;6:56.

84. Lerner UH, Persson E. Osteotropic effects by the neuropeptides calcitonin gene-related peptide, substance $\mathrm{P}$ and vasoactive intestinal peptide. J Musculoskelet Neuronal Interact. 2008;8:154-65.

85. Hohmann EL, Elde RP, Rysavy JA, Einzig S, Gebhard RL. Innervation of periosteum and bone by sympathetic vasoactive intestinal peptide-containing nerve fibers. Science. 1986;232:868-71.

86. Bataille C, Mauprivez C, Hay E, Baroukh B, Brun A, Chaussain C, et al. Different sympathetic pathways control the metabolism of distinct bone envelopes. Bone. 2012;50:1162-72.

87. Suzuki A, Kotoyori J, Oiso Y, Kozawa O. Pituitary adenylate cyclase-activating polypeptide induces cAMP production independently from vasoactive intestinal polypeptide in osteoblastlike cells. Cell Signal. 1994;6:11-6.

88. Onuoha GN. Circulating sensory peptide levels within $24 \mathrm{~h}$ of human bone fracture. Peptides. 2001;22:1107-10.

89. Ma W, Zhang X, Shi S, Zhang Y. Neuropeptides stimulate human osteoblast activity and promote gap junctional intercellular communication. Neuropeptides. 2013;47:179-86.

90. Lundberg P, Lundgren I, Mukohyama H, Lehenkari PP, Horton MA, Lerner UH. Vasoactive intestinal peptide (VIP)/pituitary adenylate cyclase-activating peptide receptor subtypes in mouse calvarial osteoblasts: presence of VIP-2 receptors and differentiation-induced expression of VIP-1 receptors. Endocrinology. 2001;142:339-47.

91. Okura H, Sato S, Kishikawa S, Kaneto S, Nakashima T, Yoshida N, et al. Runx2-I isoform contributes to fetal bone formation even in the absence of specific N-terminal amino acids. PLoS One. 2014;9:e108294. 
92. Li TF, Dong Y, Ionescu AM, Rosier RN, Zuscik MJ, Schwarz EM, et al. Parathyroid hormonerelated peptide (PTHrP) inhibits Runx2 expression through the PKA signaling pathway. Exp Cell Res. 2004;299:128-36.

93. Duan Y, Wang Z, Zhang H, He Y, Fan R, Cheng Y, et al. Extremely low frequency electromagnetic field exposure causes cognitive impairment associated with alteration of the glutamate level, MAPK pathway activation and decreased CREB phosphorylation in mice hippocampus: reversal by procyanidins extracted from the lotus seedpod. Food Funct. 2014;5:2289-97.

94. Pitts RL, Wang S, Jones EA, Symes AJ. Transforming growth factor-beta and ciliary neurotrophic factor synergistically induce vasoactive intestinal peptide gene expression through the cooperation of Smad, STAT, and AP-1 sites. J Biol Chem. 2001;276:19966-73.

95. Persson E, Lerner UH. The neuropeptide VIP regulates the expression of osteoclastogenic factors in osteoblasts. J Cell Biochem. 2011;112:3732-41.

96. Li D, Jiao J, Shatos MA, Hodges RR, Dartt DA. Effect of VIP on intracellular [Ca2+], extracellular regulated kinase $1 / 2$, and secretion in cultured rat conjunctival goblet cells. Invest Ophthalmol Vis Sci. 2013;54:2872-84.

97. Mustafa T, Grimaldi M, Eiden LE. The hop cassette of the PAC1 receptor confers coupling to $\mathrm{Ca} 2+$ elevation required for pituitary adenylate cyclase-activating polypeptide-evoked neurosecretion. J Biol Chem. 2007;282:8079-91.

98. Maeda Y, Sekiguchi F, Yamanaka R, Sugimoto R, Yamasoba D, Tomita S, et al. Mechanisms for proteinase-activated receptor 1-triggered prostaglandin E2 generation in mouse osteoblastic MC3T3-E1 cells. Biol Chem. 2015;396:153-62.

99. Lv B, Tang Y, Chen F, Xiao X. Vasoactive intestinal peptide and pituitary adenylate cyclaseactivating polypeptide inhibit tissue factor expression in monocyte in vitro and in vivo. Shock. 2009;31:185-91.

100. Miraoui H, Oudina K, Petite H, Tanimoto Y, Moriyama K, Marie PJ. Fibroblast growth factor receptor 2 promotes osteogenic differentiation in mesenchymal cells via ERK1/2 and protein kinase C signaling. J Biol Chem. 2009;284:4897-904.

101. May V, Clason TA, Buttolph TR, Girard BM, Parsons RL. Calcium influx, but not intracellular calcium release, supports PACAP-mediated ERK activation in HEK PAC1 receptor cells. J Mol Neurosci. 2014;54:342-50.

102. Hayashi K, Endoh T, Shibukawa Y, Yamamoto T, Suzuki T. VIP and PACAP inhibit L-, N- and $\mathrm{P} / \mathrm{Q}$-type $\mathrm{Ca} 2+$ channels of parasympathetic neurons in a voltage independent manner. Bull Tokyo Dent Coll. 2002;43:31-9.

103. Harfi I, Sariban E. Mechanisms and modulation of pituitary adenylate cyclase-activating protein-induced calcium mobilization in human neutrophils. Ann N Y Acad Sci. 2006;1070:322-9.

104. Payet MD, Bilodeau L, Breault L, Fournier A, Yon L, Vaudry H, et al. PAC1 receptor activation by PACAP-38 mediates $\mathrm{Ca} 2+$ release from a cAMP-dependent pool in human fetal adrenal gland chromaffin cells. J Biol Chem. 2003;278:1663-70.

105. Akopian A, Demulder A, Ouriaghli F, Corazza F, Fondu P, Bergmann P. Effects of CGRP on human osteoclast-like cell formation: a possible connection with the bone loss in neurological disorders? Peptides. 2000;21:559-64.

106. Winding B, Wiltink A, Foged NT. Pituitary adenylyl cyclase-activating polypeptides and vasoactive intestinal peptide inhibit bone resorption by isolated rabbit osteoclasts. Exp Physiol. 1997;82:871-86.

107. Franceschi RT, Xiao G. Regulation of the osteoblast-specific transcription factor, Runx2: responsiveness to multiple signal transduction pathways. J Cell Biochem. 2003;88:446-54.

108. Park HJ, Baek K, Baek JH, Kim HR. The cooperation of CREB and NFAT is required for PTHrP-induced RANKL expression in mouse osteoblastic cells. J Cell Physiol. 2015;230:667-79. 